University of Nebraska - Lincoln

DigitalCommons@University of Nebraska - Lincoln

Nebraska Beef Cattle Reports

Animal Science Department

2016

\title{
Metabolic and Body Temperature Responses to Environmental Conditions across Seasons in Finishing Steers
}

\author{
Bradley M. Boyd \\ University of Nebraska-Lincoln, bboyd4@unl.edu \\ Terry L. Mader \\ University of Nebraska-Lincoln, tmader1@unl.edu \\ Curtis J. Bittner Bittner \\ University of Nebraska-Lincoln, curtis.bittner@unl.edu \\ Dirk Burken \\ University of Nebraska-Lincoln, dburken2@unl.edu \\ Henry Hilscher \\ University of Nebraska-Lincoln \\ See next page for additional authors
}

Follow this and additional works at: https://digitalcommons.unl.edu/animalscinbcr

Part of the Meat Science Commons

Boyd, Bradley M.; Mader, Terry L.; Bittner, Curtis J. Bittner; Burken, Dirk; Hilscher, Henry; Wijffels, Gene; Gaughan, John B.; Sullivan, Megan; Cawdell- Smith, Judy; and Erickson, Galen E., "Metabolic and Body Temperature Responses to Environmental Conditions across Seasons in Finishing Steers" (2016). Nebraska Beef Cattle Reports. 861.

https://digitalcommons.unl.edu/animalscinbcr/861

This Article is brought to you for free and open access by the Animal Science Department at DigitalCommons@University of Nebraska - Lincoln. It has been accepted for inclusion in Nebraska Beef Cattle Reports by an authorized administrator of DigitalCommons@University of Nebraska - Lincoln. 


\section{Authors}

Bradley M. Boyd, Terry L. Mader, Curtis J. Bittner Bittner, Dirk Burken, Henry Hilscher, Gene Wijffels, John B. Gaughan, Megan Sullivan, Judy Cawdell- Smith, and Galen E. Erickson 


\title{
Metabolic and Body Temperature Responses to Environmental Conditions across Seasons in Finishing Steers
}

\author{
Bradley M. Boyd, Terry L. Mader, Curt Bittner, Dirk Burken, Henry Hilscher, \\ Gene Wijffels, John B. Gaughan, Megan Sullivan, Judy Cawdell-Smith and Galen E. Erickson
}

\begin{abstract}
Summary
Two trials (summer and winter) were conducted with 80 steers each at the UNL ARDC near Mead, Neb. Continuous body temperature was collected throughout both trials and blood samples were taken every other week until four weeks prior to harvest. This resulted in a total of 6 collections for the summer and 8 collections for the winter. Blood measures responded differently between seasons with significant quadratic interactions between collection time and season suggesting environmental conditions affect blood metabolites.
\end{abstract}

\section{Introduction}

Environmental stress can cause substantial economic losses to producers through both losses in animal performance and mortality. Summer conditions consisting of above normal ambient temperature, relative humidity, and solar radiation coupled with low wind speeds can increase an animal's heat load resulting in reduced performance, decreased animal comfort, and death (Journal of Animal Science, 84:712719). In addition, cold stress can result in sustained performance losses, particularly when coupled with wet and/or windy conditions. Even though environmental stress has been a researched topic for the past few decades little is known about how hot and cold environmental temperatures affect cattle metabolically, and whether significant interactions exists among seasons (hot vs cold) for various physiological parameters. Additionally, even though body temperature has been measured, measures have been recorded over only short periods of time. Therefore, the objective of the current studies was to determine the effect of season and ambient temperature on animal blood parameters in addition to body temperature measured continuously throughout the feeding period.

(c) The Board Regents of the University of Nebraska. All rights reserved.

\section{Procedure}

Crossbred beef steers were utilized to conduct two studies (80 steers, 10 steers/ pen) at the University of Nebraska Agricultural Research and Development Center research feedlot near Mead, Nebraska. Cattle in both experiments were limit fed a diet consisting of 50\% Sweet Bran (Cargill, Blair, Neb) and $50 \%$ alfalfa hay at an estimated $2 \%$ of BW for five days prior to an initial BW being collected over $2 \mathrm{~d}$. The first trial was conducted during the summer utilizing summer yearlings (initial BW $=1076 \pm 45 \mathrm{lb}$ ). Cattle were started on trial May 22, 2014 and harvested on September 10, 2014 (summer). Fall yearlings (initial $\mathrm{BW}=853 \pm 35 \mathrm{lb}$ ) were used for the second trial and fed during the fall and winter with cattle starting on feed on September 11, 2014 and harvested on January 14, 2015 (winter). Cattle in both trials were stepped onto the finishing diet over a 21 day period by reducing alfalfa inclusion in the diet and increasing levels of HMC. Cattle in both trials were fed the same finishing diet consisting of $40 \%$ Sweet Bran (Cargill, Blair, $\mathrm{Neb),51 \%} \mathrm{HMC,} \mathrm{5 \%} \mathrm{wheat} \mathrm{straw,} \mathrm{and} 4 \%$ supplement.

On the first day of both trials, blood samples were collected via jugular venous puncture from each steer to obtain a baseline measure. Blood samples were collected from every steer in two week intervals until 4 weeks prior to harvest, resulting in 6 blood collections for the summer trial and 8 collections for the winter trial. Three separate blood samples were collected from each steer during each collection. One sample was allowed to clot and was then centrifuged in the lab to separate blood cells from blood serum and samples were sent to Nebraska Lab-Link (Lincoln, Neb) for analysis. A second sample was collected using EDTA containing vacutainers and sent to Physicians Lab (Omaha, Neb) for analysis. The final sample was centrifuged in the lab and blood plasma was removed and plasma samples were sent to Commonwealth Scientific and Industrial Research
Organisation (CSIRO; Queensland, Aus)

for subsequent analysis.

All cattle in both trials received a SmartStock (SmartStock; LLC. Pawnee, OK) temperature monitoring rumen bolus at the initiation of the trial. These boluses transmitted each individual steer's body temperature in ten minute intervals (summer) and twenty minute intervals (winter) to a central computer where data were compiled across days. Any missing data points were removed and average body temperature was calculated for every hour creating a final data set with individual animal body temperatures every hour throughout the duration of the finishing period.

Blood data, and body temperature were correlated to environmental temperature and comprehensive climate index (CCI) using the CORR procedure of SAS (SAS Institute, Inc. Cary, N.C.) with steer as the experimental unit. Pen intakes (DM offered) were also correlated to environmental temperature and CCI using the CORR procedure of SAS (SAS Institute, Inc. Carry, N. C.) with pen as the experimental unit. Linear and quadratic relationships between blood collection time and season were analyzed using the GLIMMIX procedure of SAS with steer as the experimental unit. The model included the fixed effects of time, season, and the linear and quadratic interactions of time and season.

\section{Results}

Only blood measures that had a correlation coefficient of 0.40 or greater to at least one of the 3-d, 7-d, or 14-d average CCI or ambient temperature were chosen to be reported. Environmental temperature data for both trials related to blood collections are presented in Table 1. Bilirubin, a powerful antioxidant (The International Journal of Biochemistry \& Cell Biology 34:216-220), was positively correlated ( $\mathrm{r}>$ 0.71 ; Table 2) with CCI measured for the previous 3-d, 7-d, and 14-d averages for the 
winter trial, but was negatively correlated $(\mathrm{r}<-0.13)$ for the previous $3-\mathrm{d}, 7-\mathrm{d}$, and 14- $d$ averages for the summer trial. This opposite correlations suggest that cattle physiology may react differently to hot and cold environmental temperatures. Glucose, mean corpuscular hemoglobin $(\mathrm{MCH})$, mean corpuscular volume (MCV), and sodium levels were also correlated differently between the summer and winter trials. For the 7- $\mathrm{d}$ and 14- $\mathrm{d}$ average CCI and environmental temperature, glucose, $\mathrm{MCH}$, and MCV levels were positively correlated ( $r$ > 0.19 ) for the summer trial but negatively correlated $(\mathrm{r}<-0.38)$ for the winter trial (Table 2). However, when correlated to the 3-d average glucose, MCH, and MCV levels were slightly negatively correlated $(\mathrm{r}<$

-0.08 ) to CCI and environmental temperature for both summer and winter trials. Sodium levels, in the summer trial, were positively correlated $(r>0.48)$ to the $3-\mathrm{d}$, 7-d, and 14- $\mathrm{d}$ average CCI and environmental temperature; however, in the winter, sodium levels were negatively correlated ( $\mathrm{r}$ $<0.30$; Table 2).

Hemoglobin and hematocrit were both negatively correlated $(r>-0.35)$ to the $3-d$, 7-d, and 14- $\mathrm{d}$ average CCI and environmental temperature for both summer and winter trials. This is supported by previous findings where a negative correlation between hematocrit levels and ambient temperature in dairy cattle was reported (Journal of Dairy Science 59:104-108). It has been noted that there are significant effects of ambient temperature on hemoglobin levels when dairy cows were exposed to cool, intermediate, and hot environments (Journal of Dairy Science 64:62-70). This was attributed to a decrease in cellular oxygen requirements to reduce the heat load of the animal (Journal of Dairy Science 64:62-70) supporting similar findings where it was suggested that the oxygen binding capacity of blood is decreased under heat stress (Journal of Dairy Science 59:104-108).

Season had a significant effect on all blood parameters $(P \leq 0.04)$ with the exception of creatine kinase levels and blood urea nitrogen: creatine ratio $(P \geq 0.22$; Table 3). Additionally, all blood measures had a significant quadratic interaction between season and collection $(P \leq 0.03)$ with the exception of $\mathrm{CO}_{2}(P=0.67$; Table $3)$ which had a linear interaction $(P<$

Table 1. Environmental temperature $\left({ }^{\circ} \mathrm{F}\right)$ and comprehensive climate index (CCI) averages based on blood collections

\begin{tabular}{|c|c|c|c|c|c|c|c|}
\hline \multirow[t]{2}{*}{ Collection $^{\mathrm{a}}$} & \multirow[t]{2}{*}{ Season $^{b}$} & \multicolumn{2}{|c|}{ Three Day Avg } & \multicolumn{2}{|c|}{ Seven Day Avg ${ }^{\mathrm{d}}$} & \multicolumn{2}{|c|}{ Fourteen Day Avg } \\
\hline & & CCI & Temp $^{f}$ & CCI & Temp $^{f}$ & CCI & Temp $^{f}$ \\
\hline \multirow[t]{2}{*}{1} & $\mathrm{~S}$ & 72.36 & 72.18 & 60.36 & 60.97 & 57.07 & 59.34 \\
\hline & $\mathrm{W}$ & 66.53 & 66.97 & 67.58 & 65.80 & 73.11 & 68.76 \\
\hline \multirow[t]{2}{*}{2} & S & 75.46 & 70.24 & 77.96 & 73.26 & 77.17 & 71.95 \\
\hline & $\mathrm{W}$ & 66.63 & 64.72 & 68.55 & 66.20 & 64.25 & 62.29 \\
\hline \multirow[t]{2}{*}{3} & $\mathrm{~S}$ & 81.24 & 81.11 & 74.30 & 74.44 & 72.93 & 71.45 \\
\hline & W & 59.53 & 59.65 & 51.51 & 54.38 & 61.14 & 60.72 \\
\hline \multirow[t]{2}{*}{4} & $\mathrm{~S}$ & 69.48 & 67.14 & 73.92 & 70.79 & 78.07 & 72.74 \\
\hline & $\mathrm{W}$ & 58.82 & 59.28 & 55.12 & 56.37 & 51.60 & 54.05 \\
\hline \multirow[t]{2}{*}{5} & $\mathrm{~S}$ & 70.42 & 64.96 & 78.35 & 71.42 & 79.84 & 72.59 \\
\hline & $\mathrm{W}$ & 44.49 & 50.87 & 39.08 & 47.03 & 47.45 & 51.36 \\
\hline \multirow[t]{2}{*}{6} & $\mathrm{~S}$ & 74.87 & 67.85 & 79.82 & 73.15 & 80.21 & 74.37 \\
\hline & $\mathrm{W}$ & 14.53 & 23.22 & 9.04 & 20.08 & 15.23 & 26.41 \\
\hline \multirow[t]{2}{*}{7} & S & - & - & - & - & - & - \\
\hline & $\mathrm{W}$ & 16.99 & 24.95 & 18.99 & 28.95 & 20.70 & 32.01 \\
\hline \multirow[t]{2}{*}{8} & $\mathrm{~S}$ & - & - & - & - & - & - \\
\hline & $\mathrm{W}$ & 12.42 & 26.55 & 27.35 & 38.43 & 25.27 & 35.55 \\
\hline
\end{tabular}

${ }^{a}$ Summer collections taken every other week beginning on 5/22/2014 and ending 7/31/2014. Winter collections taken every other week beginning on 9/11/2014 and ending on 12/18/2014.

${ }^{\mathrm{b}}$ Season either defined as $\mathrm{S}=$ Summer or $\mathrm{W}=$ Winter.

'Three days prior to blood collection weather averages correlated to blood measures.

dSeven days prior to blood collection weather averages correlated to blood measures.

${ }^{e}$ Fourteen days prior to blood collection weather averages correlated to blood measures.

collection weather averages correlated to blood measures

${ }^{\mathrm{f}}$ Environmental Temperature ${ }^{\circ} \mathrm{F}$

0.001) between season and collection. This suggests that the blood parameters changed quadratically across collection periods for both the summer and winter trials; however, the quadratic response to collection is not the same between seasons suggesting the change in these parameters is due to environmental conditions rather than an effect due to days on feed or BW.

There was a sharp decrease observed for bilirubin, blood urea nitrogen (BUN), and creatine kinase during the summer between the first and second blood collection followed by a leveling off for the remaining collections during the summer (Table 3 ). This sharp decrease occurred during a time where the 7- $\mathrm{d}$ average CCI increased from 60.36 to 77.96 followed by a leveling off. The sudden decrease and then level period in these blood measures suggests steers metabolically adapt to changing temperature over time. Furthermore, during the winter trial a sharp increase in bilirubin, BUN, and creatine kinase was observed between the 5th and 6th blood collections. This sharp increase occurred during a time where the 7 - $d$ average CCI increased from 9.04 to 18.99 . This sharp increase suggests that animals were adapted to very cold conditions and when conditions warmed the animal attempted to compensate thus a large increase in these blood measures was observed. Other studies have suggested that the acclimation period to environmental conditions for most cattle is between 9-14 days (Animal 4:1167-1183). This supports the findings of the current study that suggests that the levels of bilirubin, BUN, and creatine kinase leveled off in the summer trial by the 3 rd blood collection which was 14 days after the spike was observed during the 2 nd blood collection.

Ruminal temperature was positively correlated $(r>0.12)$ to the previous day average CCI and environmental temperature in the summer trial (Table 4). This suggests that the previous day environmental conditions impacted body tempera- 
Table 2. Correlation between environmental temperature and the comprehensive climate index (CCI) to blood measures

\begin{tabular}{|c|c|c|c|c|c|c|c|c|c|c|c|c|c|}
\hline \multirow[t]{2}{*}{ Blood Measure } & \multirow[t]{2}{*}{ Season $^{\mathrm{a}}$} & \multicolumn{4}{|c|}{ Three day ${ }^{\mathrm{b}}$} & \multicolumn{4}{|c|}{ Seven Day } & \multicolumn{4}{|c|}{ Fourteen Day } \\
\hline & & $\mathrm{CCI}^{\mathrm{e}}$ & $P$-value & Temp $^{f}$ & $P$-value & $\mathrm{CCI}^{\mathrm{e}}$ & $P$-value & $\mathrm{Temp}^{\mathrm{f}}$ & $P$-value & $\mathrm{CCI}^{\mathrm{e}}$ & $P$-value & Temp $^{f}$ & $P$-value \\
\hline \multirow[t]{2}{*}{ Hemoglobin, g/dL } & S & -0.30 & $<0.01$ & -0.25 & $<0.01$ & -0.30 & $<0.01$ & -0.44 & $<0.01$ & -0.29 & $<0.01$ & -0.35 & $<0.01$ \\
\hline & W & -0.57 & $<0.01$ & -0.54 & $<0.01$ & -0.51 & $<0.01$ & -0.49 & $<0.01$ & -0.51 & $<0.01$ & -0.50 & $<0.01$ \\
\hline \multirow[t]{2}{*}{ Hematocrit, \% } & S & -0.30 & $<0.01$ & -0.22 & $<0.01$ & -0.35 & $<0.01$ & -0.48 & $<0.01$ & -0.34 & $<0.01$ & -0.40 & $<0.01$ \\
\hline & $\mathrm{W}$ & -0.59 & $<0.01$ & -0.57 & $<0.01$ & -0.54 & $<0.01$ & -0.52 & $<0.01$ & -0.54 & $<0.01$ & -0.54 & $<0.01$ \\
\hline \multirow[t]{2}{*}{$\mathrm{MCV}^{\mathrm{g}}, \mathrm{L} /$ cell } & S & -0.17 & $<0.01$ & -0.26 & $<0.01$ & 0.28 & $<0.01$ & 0.19 & $<0.01$ & 0.33 & $<0.01$ & 0.31 & $<0.01$ \\
\hline & $\mathrm{W}$ & -0.40 & $<0.01$ & -0.40 & $<0.01$ & -0.42 & $<0.01$ & -0.40 & $<0.01$ & -0.43 & $<0.01$ & -0.42 & $<0.01$ \\
\hline \multirow[t]{2}{*}{$\mathrm{MCH}^{\mathrm{h}}, \mathrm{g} /$ cell } & S & -0.14 & 0.01 & -0.27 & $<0.01$ & 0.33 & 0.01 & 0.24 & $<0.01$ & 0.37 & $<0.01$ & 0.35 & $<0.01$ \\
\hline & $\mathrm{W}$ & -0.37 & $<0.01$ & -0.37 & $<0.01$ & -0.38 & $<0.01$ & -0.37 & $<0.01$ & -0.39 & $<0.01$ & -0.38 & $<0.01$ \\
\hline \multirow[t]{2}{*}{ Eosinophils,\% } & S & -0.13 & 0.01 & -0.29 & $<0.01$ & 0.43 & 0.01 & 0.35 & $<0.01$ & 0.46 & $<0.01$ & 0.42 & $<0.01$ \\
\hline & $\mathrm{W}$ & -0.07 & 0.09 & -0.06 & 0.12 & -.077 & 0.05 & -0.07 & 0.08 & -0.07 & 0.08 & -0.07 & 0.10 \\
\hline \multirow[t]{2}{*}{$\mathrm{A} / \mathrm{G}$, ratio } & S & 0.02 & 0.67 & 0.01 & 0.79 & -0.32 & $<0.01$ & -0.37 & $<0.01$ & -0.42 & $<0.01$ & -0.46 & $<0.01$ \\
\hline & $\mathrm{W}$ & 0.30 & $<0.01$ & 0.30 & $<0.01$ & 0.39 & $<0.01$ & 0.39 & $<0.01$ & 0.31 & $<0.01$ & 0.32 & $<0.01$ \\
\hline \multirow[t]{2}{*}{ Albumin, g/dL } & S & -0.05 & 0.27 & -0.06 & 0.18 & -0.35 & $<0.01$ & -0.44 & $<0.01$ & -0.45 & $<0.01$ & -0.50 & $<0.01$ \\
\hline & $\mathrm{W}$ & 0.03 & 0.53 & 0.01 & 0.75 & 0.08 & 0.03 & 0.07 & 0.10 & 0.03 & 0.44 & 0.03 & 0.49 \\
\hline \multirow[t]{2}{*}{$\mathrm{BC}^{\mathrm{i}}$, ratio } & S & -0.18 & $<0.01$ & -0.04 & 0.36 & -0.61 & $<0.01$ & -0.69 & $<0.01$ & -0.67 & $<0.01$ & -0.73 & $<0.01$ \\
\hline & $\mathrm{W}$ & 0.07 & 0.08 & 0.08 & 0.04 & 0.13 & 0.01 & 0.12 & 0.01 & 0.14 & $<0.01$ & 0.13 & $<0.01$ \\
\hline \multirow[t]{2}{*}{ Bilirubin, mg/dL } & $\mathrm{S}$ & -0.13 & 0.01 & 0.01 & 0.77 & -0.32 & $<0.01$ & -0.33 & $<0.01$ & -0.30 & $<0.01$ & -0.31 & $<0.01$ \\
\hline & $\mathrm{W}$ & 0.75 & $<0.01$ & 0.73 & $<0.01$ & 0.72 & $<0.01$ & 0.71 & $<0.01$ & 0.74 & $<0.01$ & 0.73 & $<0.01$ \\
\hline \multirow[t]{2}{*}{ BUN', mg/dL } & $\mathrm{S}$ & -0.15 & 0.01 & -0.04 & 0.43 & -0.59 & $<0.01$ & -0.67 & $<0.01$ & -0.65 & $<0.01$ & -0.69 & $<0.01$ \\
\hline & $\mathrm{W}$ & 0.10 & 0.01 & 0.11 & 0.01 & 0.18 & $<0.01$ & 0.17 & $<0.01$ & 0.19 & $<0.01$ & 0.18 & $<0.01$ \\
\hline \multirow{2}{*}{$\begin{array}{l}\text { Creatine kinase, } \\
\mathrm{U} / \mathrm{L}\end{array}$} & $\mathrm{S}$ & -0.12 & 0.01 & 0.04 & 0.35 & -0.48 & $<0.01$ & -0.49 & $<0.01$ & -0.49 & $<0.01$ & -0.51 & $<0.01$ \\
\hline & $\mathrm{W}$ & 0.07 & 0.07 & 0.08 & 0.04 & 0.12 & 0.01 & 0.12 & 0.01 & 0.11 & 0.01 & 0.11 & 0.01 \\
\hline \multirow[t]{2}{*}{$\mathrm{CO}_{2}, \mathrm{mM} / \mathrm{dL}$} & $\mathrm{S}$ & -0.04 & 0.35 & -0.32 & $<0.01$ & 0.64 & $<0.01$ & 0.54 & $<0.01$ & 0.66 & $<0.01$ & 0.64 & $<0.01$ \\
\hline & $\mathrm{W}$ & 0.24 & $<0.01$ & 0.27 & $<0.01$ & 0.28 & $<0.01$ & 0.29 & $<0.01$ & 0.29 & $<0.01$ & 0.29 & $<0.01$ \\
\hline \multirow[t]{2}{*}{ Glucose, mg/dL } & S & -0.08 & 0.08 & -0.16 & $<0.01$ & 0.35 & $<0.01$ & 0.34 & $<0.01$ & 0.41 & $<0.01$ & 0.41 & $<0.01$ \\
\hline & $\mathrm{W}$ & -0.37 & $<0.01$ & -0.37 & $<0.01$ & -0.42 & $<0.01$ & -0.42 & $<0.01$ & -0.42 & $<0.01$ & -0.42 & $<0.01$ \\
\hline \multirow[t]{2}{*}{ Sodium, mEq/L } & $\mathrm{S}$ & 0.09 & 0.05 & -0.15 & 0.01 & 0.54 & $<0.01$ & 0.48 & $<0.01$ & 0.53 & $<0.01$ & 0.53 & $<0.01$ \\
\hline & $\mathrm{W}$ & -0.30 & $<0.01$ & -0.33 & $<0.01$ & -0.35 & $<0.01$ & -0.37 & $<0.01$ & -0.41 & $<0.01$ & -0.41 & $<0.01$ \\
\hline
\end{tabular}

a Season either defined as $\mathrm{S}=$ Summer or $\mathrm{W}=$ Winter.

${ }^{\text {b}}$ Three days prior to blood collection weather averages correlated to blood measures.

'Seven days prior to blood collection weather averages correlated to blood measures.

dFourteen days prior to blood collection weather averages correlated to blood measures.

'From Journal of Animal Science 88:2153-2165.

${ }^{\mathrm{f}} \mathrm{Temp}=$ Environmental Temperature ${ }^{\circ} \mathrm{F}$

${ }^{\mathrm{g}} \mathrm{MCV}=$ mean corpuscular volume.

${ }^{\mathrm{h}} \mathrm{MCH}=$ Mean Corpuscular Hemoglobin

${ }^{\mathrm{B}} \mathrm{BC}=$ Blood urea nitrogen: creatine ratio.

${ }^{j}$ BUN $=$ Blood Urea Nitrogen. 
Table 3: Linear and Quadratic relationship between bleed date and season

\begin{tabular}{|c|c|c|c|c|c|c|c|c|c|c|c|c|c|c|}
\hline \multirow{2}{*}{$\begin{array}{l}\text { Blood } \\
\text { Measure }\end{array}$} & \multirow[t]{2}{*}{ Season $^{\mathrm{b}}$} & \multicolumn{9}{|c|}{ Collection $^{\mathrm{a}}$} & \multicolumn{4}{|c|}{$P$-value } \\
\hline & & 1 & 2 & 3 & 4 & 5 & 6 & 7 & 8 & SEM & $\mathrm{S}^{\mathrm{c}}$ & $\mathrm{C}^{\mathrm{d}}$ & $\mathrm{C}^{*} \mathrm{~S}^{\mathrm{e}}$ & $\begin{array}{l}C * C \\
* S^{f}\end{array}$ \\
\hline \multirow{2}{*}{$\begin{array}{l}\text { Hemoglobin, } \\
\text { g/dL }\end{array}$} & S & 12.47 & 12.96 & 13.51 & 14.02 & 14.18 & 14.68 & - & - & 0.11 & $<0.01$ & 0.43 & 0.53 & $<0.01$ \\
\hline & $\mathrm{W}$ & 14.79 & 13.51 & 12.89 & 13.56 & 13.87 & 14.16 & 13.44 & 11.96 & & & & & \\
\hline \multirow{2}{*}{$\begin{array}{l}\text { Hematocrit, } \\
\%\end{array}$} & S & 34.52 & 35.99 & 37.64 & 39.04 & 39.76 & 40.62 & - & - & 0.31 & $<0.01$ & $<0.01$ & 0.04 & $<0.01$ \\
\hline & $\mathrm{W}$ & 42.49 & 38.53 & 36.61 & 38.63 & 39.23 & 40.01 & 36.75 & 33.33 & & & & & \\
\hline \multirow[t]{2}{*}{$\mathrm{MCV}^{\mathrm{f}}, \mathrm{L} /$ cell } & $S$ & 44.47 & 45.68 & 46.20 & 46.41 & 46.84 & 46.72 & - & - & 0.36 & $<0.01$ & $<0.01$ & $<0.01$ & $<0.01$ \\
\hline & $\mathrm{W}$ & 43.25 & 43.35 & 44.63 & 46.33 & 47.34 & 47.41 & 41.54 & 43.22 & & & & & \\
\hline \multirow[t]{2}{*}{$\mathrm{MCH}^{\mathrm{h}}, \mathrm{g} /$ cell } & S & 16.04 & 16.43 & 16.55 & 16.65 & 16.69 & 16.89 & - & - & 0.13 & $<0.01$ & $<0.01$ & $<0.01$ & $<0.01$ \\
\hline & $\mathrm{W}$ & 15.03 & 15.20 & 15.70 & 16.24 & 16.72 & 16.77 & 15.19 & 15.49 & & & & & \\
\hline \multirow[t]{2}{*}{ Eosinophils,\% } & $\mathrm{S}$ & 4.26 & 4.29 & 4.17 & 3.81 & 3.95 & 4.26 & - & - & 0.29 & $<0.01$ & 0.10 & 0.02 & $<0.01$ \\
\hline & $\mathrm{W}$ & 1.63 & 5.43 & 3.06 & 4.93 & 4.95 & 4.40 & 3.28 & 2.97 & & & & & \\
\hline \multirow[t]{2}{*}{ A/G, ratio } & $\mathrm{S}$ & 1.34 & 1.41 & 0.89 & 0.85 & 0.86 & 0.98 & - & - & 0.02 & 0.01 & $<0.01$ & $<0.01$ & $<0.01$ \\
\hline & $\mathrm{W}$ & 0.91 & 0.94 & 0.80 & 0.80 & 0.86 & 0.93 & 0.95 & 1.02 & & & & & \\
\hline \multirow{2}{*}{$\begin{array}{l}\text { Albumin, } \\
\text { g/dL }\end{array}$} & S & 4.31 & 4.29 & 3.47 & 3.47 & 3.55 & 3.78 & - & - & 0.03 & 0.04 & $<0.01$ & $<0.01$ & $<0.01$ \\
\hline & $\mathrm{W}$ & 3.53 & 3.65 & 3.39 & 3.58 & 3.69 & 3.62 & 3.72 & 3.65 & & & & & \\
\hline \multirow[t]{2}{*}{$\mathrm{BC}^{\mathrm{i}}$, ratio } & $\mathrm{S}$ & 17.29 & 13.49 & 8.10 & 8.51 & 9.60 & 9.67 & - & - & 0.48 & 0.68 & 0.47 & $<0.01$ & $<0.01$ \\
\hline & $\mathrm{W}$ & 10.00 & 9.35 & 10.09 & 11.71 & 10.34 & 12.10 & 16.48 & 11.22 & & & & & \\
\hline \multirow{2}{*}{$\begin{array}{l}\text { Bilirubin, } \\
\mathrm{mg} / \mathrm{dL}\end{array}$} & S & 0.23 & 0.14 & 0.18 & 0.18 & 0.19 & 0.18 & - & - & 0.01 & $<0.01$ & $<0.01$ & $<0.01$ & $<0.01$ \\
\hline & $\mathrm{W}$ & 0.21 & 0.17 & 0.10 & 0.05 & 0.03 & 0.04 & 0.24 & 0.22 & & & & & \\
\hline \multirow[t]{2}{*}{$\mathrm{BUN}^{j}, \mathrm{mg} / \mathrm{dL}$} & $\mathrm{S}$ & 19.62 & 14.97 & 10.38 & 10.75 & 11.02 & 12.97 & - & - & 0.32 & 0.01 & $<0.01$ & $<0.01$ & $<0.01$ \\
\hline & $\mathrm{W}$ & 11.22 & 10.22 & 11.41 & 12.81 & 11.87 & 12.87 & 16.32 & 13.46 & & & & & \\
\hline \multirow{2}{*}{$\begin{array}{l}\text { Creatine } \\
\text { kinase, U/L }\end{array}$} & S & 296.82 & 189.30 & 154.15 & 164.00 & 154.15 & 145.04 & - & - & 12.88 & 0.22 & 0.77 & $<0.01$ & 0.03 \\
\hline & $\mathrm{W}$ & 160.08 & 182.84 & 175.37 & 179.74 & 177.85 & 205.99 & 258.41 & 193.23 & & & & & \\
\hline \multirow[t]{2}{*}{$\mathrm{CO}_{2}, \mathrm{mM} / \mathrm{dL}$} & S & 24.09 & 27.49 & 26.68 & 28.09 & 28.23 & 29.59 & - & - & 0.23 & $<0.01$ & $>0.01$ & $<0.01$ & 0.67 \\
\hline & $\mathrm{W}$ & 26.51 & 27.51 & 27.44 & 25.73 & 25.76 & 27.16 & 29.08 & 26.54 & & & & & \\
\hline \multirow{2}{*}{$\begin{array}{l}\text { Glucose, } \\
\mathrm{mg} / \mathrm{dL}\end{array}$} & S & 66.51 & 77.52 & 79.75 & 89.30 & 82.64 & 82.75 & - & - & 1.42 & $<0.01$ & $<0.01$ & 0.67 & $<0.01$ \\
\hline & $\mathrm{W}$ & 91.15 & 93.15 & 92.62 & 99.17 & 92.84 & 93.33 & 74.66 & 81.53 & & & & & \\
\hline \multirow{2}{*}{$\begin{array}{l}\text { Sodium, } \\
\mathrm{mEq} / \mathrm{L}\end{array}$} & S & 137.94 & 139.38 & 139.95 & 139.59 & 140.85 & 141.20 & - & - & 0.22 & $<0.01$ & 0.41 & $<0.01$ & $<0.01$ \\
\hline & $\mathrm{W}$ & 138.52 & 141.13 & 139.19 & 140.97 & 140.73 & 139.27 & 137.92 & 139.21 & & & & & \\
\hline
\end{tabular}

aSummer collections taken every other week beginning on 5/22/2014 and ending 7/31/2014. Winter collections taken every other week beginning on 9/11/2014 and ending on 12/18/2014.

bSeason either defined as $\mathrm{S}=$ Summer or $\mathrm{W}=$ Winter.

'Effect of season.

${ }^{\mathrm{d}}$ Effect of collection.

${ }^{\mathrm{e}}$ Linear interaction between collection and season.

${ }^{\mathrm{f}} \mathrm{Quadratic}$ interaction between collection and season

${ }^{\mathrm{g}} \mathrm{MCV}=$ mean corpuscular volume.

${ }^{\mathrm{h}} \mathrm{MCH}=$ Mean Corpuscular Hemoglobin.

${ }^{i} \mathrm{BC}=$ Blood urea nitrogen: creatine ratio.

${ }^{j} \mathrm{BUN}=$ Blood Urea Nitrogen. 
ture. Conversely, in the winter trial, body temperature was negatively correlated $(r$ $<-0.23$ ) to both the previous day average CCI and environmental temperature which suggests that in the winter as environmental temperature decreases, steers are compensating and increasing body temperature. Intake was negatively correlated $(\mathrm{r}=$ -0.38 ) to the previous day average CCI and environmental temperature in the summer trial and positively correlated $(r>0.19)$ in the winter trial (Table 4). This relationship between DMI and environmental measures suggest that in the summer, when CCI and environmental temperature increase, the steers consumed less feed or vice-versa. In the winter, as environmental temperature changes, DMI changes in the same manner, although the relationship is not as strong in the winter as in the summer.

In the current study it appears that finishing steers have a different metabolic reaction to hot and cold conditions. Many blood measures responded differently to collection points between the seasons which suggests environment has a greater influence on these measures than days on feed or BW. Ruminal temperature and DMI appears to be related to environmental temperature which is consistent with findings in current literature (Journal of Thermal Biology 33:12-19). Ruminal temperature was positively correlated to the previous day environmental conditions in the summer suggesting that the previous day's

Table 4: Correlation between previous day environmental temperature and comprehensive climate index (CCI) to animal body temperature and DMI

\begin{tabular}{lcccccc}
\hline \multicolumn{1}{c}{ Measure } & Season $^{\mathrm{a}}$ & Avg. value $^{\mathrm{b}}$ & $\mathrm{CCI}^{\mathrm{c}}$ & $P$-value & Temp $^{\mathrm{d}}$ & $P$-value \\
\hline $\begin{array}{l}\text { Ruminal } \\
\text { Temperature, }{ }^{\circ} \mathrm{F}\end{array}$ & $\mathrm{S}$ & 102.86 & 0.13 & $<0.001$ & 0.12 & $<0.001$ \\
& $\mathrm{~W}$ & 102.35 & -0.24 & $<0.001$ & -0.23 & $<0.001$ \\
DMI, lb & $\mathrm{S}$ & 29.55 & -0.38 & $<0.001$ & -0.38 & $<0.001$ \\
& $\mathrm{~W}$ & 29.86 & 0.19 & $<0.001$ & 0.21 & $<0.001$ \\
\hline
\end{tabular}

aSeason either defined as $\mathrm{S}=$ summer or $\mathrm{W}=$ winter.

${ }^{b}$ Overall average value for DMI and rumen temperature of cattle for summer and winter trials

'From Journal of Animal Science 88:2153-2165

${ }^{\mathrm{d}} \mathrm{Temp}=$ Correlation to daily average environmental temperature

environmental conditions can add to the potential for environmental stress. Based on the data in these 2 studies it can be concluded that hot and cold environmental conditions affect feedlot cattle differently from a metabolic standpoint. While it is still unclear what blood metabolites are the most important during times of hot and cold environmental conditions, it is evident that blood metabolites change during periods of warm and cold weather. It appears that steers have the ability to adapt to environmental conditions over time. Therefore, sudden swings in environmental conditions may subject animals to increased stress if they have not been previously acclimated.

Bradley M. Boyd, graduate student

Terry L. Mader, professor emeritus, University of Nebraska-Lincoln Department of Animal Science
Curt Bittner, research technician

Dirk Burken, former feedlot manager

Henry Hilscher, feedlot manager

Gene Wijffels, senior research scientist, CSIRO, Queensland, Australia

John B. Gaughan, associate professor, University of Queensland, Queensland Australia

Megan Sullivan, postdoctoral research fellow, University of Queensland, Queensland, Australia

Judy Cawdell-Smith, Senior Lecturer, University of Queensland, Queensland Australia

Galen E. Erickson, professor, University of Nebraska-Lincoln Department of Animal Science, Lincoln NE 